\title{
Reframing infection control
} approaches in low-resource health care settings: A nod to the emic perspective

\section{Samantha J Sadler ${ }^{1,2,3}$, Anthony T Fuller ${ }^{1,4}$}

\author{
${ }^{1}$ Duke Global Neurosurgery \& Neurology, Durham, North Carolina, USA \\ ${ }^{2}$ Harvard Medical School, Boston, Massachusetts, USA \\ ${ }^{3}$ Duke University, Durham, North Carolina, USA \\ ${ }^{4}$ Duke University, Department of Neurosurgery, Durham, North Carolina, USA
}

$\mathrm{H}$ ealthcare-associated infections (HCAIs) comprise a significant public health concern that, like many of the world's communicable diseases, preys on the most vulnerable. Compared to highincome countries (HICs), low- and middle-income countries (LMICs) are vulnerable to higher HCAI rates, which are associated with worse patient outcomes and excess costs [1].

The recent outbreak of Severe Acute Respiratory Syndrome-Coronavirus-2 (SARS-CoV-2) threatens to exacerbate these inequities, with LMICs facing contextual challenges to controlling the virus that may limit the effectiveness of recommended approaches [2] For example, although early studies have suggested infection control efforts such

Although local infection control teams have been deemed important, their effectiveness is limited by "one-size-fits-all" guidelines broadly assigned for low-resource settings, which often lack attention to important inter-setting diversity. as early quarantine efforts may be effective in slowing the spread of this global pandemic [3], the ability to comply with these strict rules often hinges upon financial and/or structural privileges of individuals and/or institutions. This is especially true for health care institutions in LMICs that may have limited access to the recommended equipment for preventing or slowing the spread of SARS-CoV-2 within their facilities. As heightened HCAI rates constitute a major, multifaceted burden in LMICs, SARS-CoV-2 highlights how improving infection control practices in LMIC health care settings is essential to reducing infections, alleviating the financial burden on vulnerable, economically developing LMIC health care systems, and ultimately achieving more equitable outcomes [1].

\section{REFRAMING INFECTION CONTROL APPROACHES IN LOW- AND MIDDLE- INCOME SETTINGS}

As studies begin producing recommendations for controlling SARS-CoV-2 in low-resource settings, it should be recognized that effective implementation of evidence-based infection control practices remains a challenge. In addition to inadequate financial and material resources, a combination of overcrowding, understaffing and weak governance structures can render the execution of proper infection control techniques infeasible $[1,4]$.

Tailoring infection control or other quality improvement interventions may enhance project impact $[4,5]$. Gardam and colleagues have explored how local, context-specific social and cultural norms can shape 
the efficacy of an infection control intervention; moreover, choosing an infection control strategy that emphasizes local norms may foster better outcomes [6] A review of efforts to implement WHO clinical guidelines for hospital care in LMICs likewise correlated efforts to adapt the clinical guidelines to the local setting with improved implementation results [5]. Therefore, in addition to respecting broader, systemic limitations of an LMIC setting, both the intervention design and implementation methods may benefit from contextual adaptation at the local level.

Therefore, in determining how to most effectively navigate these challenges to producing effective infection control strategies in LMIC settings, researchers should (first) adopt a locally-centered conceptual

Through implementation strategies prioritizing local stakeholder empowerment, the local infection control team model could be adapted to facilitate locally informed (and thereby emic) infection control approaches. framework to ensure the foundation of the approach is culturally- and contextually-informed, (second) identify evidencebased implementation strategies that reflect the goals of the locally-centered conceptual framework, and then (third) identify an implementation model to effectively apply those evidence-based, locally-centered strategies in the context of infection control practices (Figure 1).

\section{Framework: the emic perspective}

Pike (1967) coined two different anthropological perspectives in seeking to understand context with respect to culture: the emic perspective, which emphasizes studying behavior from within the given culture itself, vs the etic perspective, which involves studying behavior from an external, alien viewpoint outside of the given culture (Pike, as cited in Berry (1989); see for a more detailed explanation) [7] In other words, the emic perspective "clarifies intrinsic cultural distinctions," whereas the etic perspective "seeks objectivity as an outside observer across cultures" [8].

This dichotomy can be applied to intervention approaches: creating an intervention that, at its core, prioritizes local application involves taking an emic approach, one which is wrought from within the perspective of the local culture, rather than an etic perspective, which is one generated from an outside perspective and applied to an alternative culture. In this way, emic, locally driven approaches have the benefit of greater contextual and cultural compatibility, which - as described here - may be important to effectively implementing interventions across different contexts.

\section{Strategies: community-based participatory research}

By definition, local stakeholder involvement and authority is tied to facilitating an emic perspective. To properly inform local adaptation, studies have called for active local stakeholder engagement in the design and implementation of infection control projects [9] Community-based participatory research (CBPR) is a good example of systematizing this; key to CBPR is an equal sharing of responsibility between re-

COMMUNITY-CENTERED

APPROACHES
INFECTION CONTROL CONTEXT

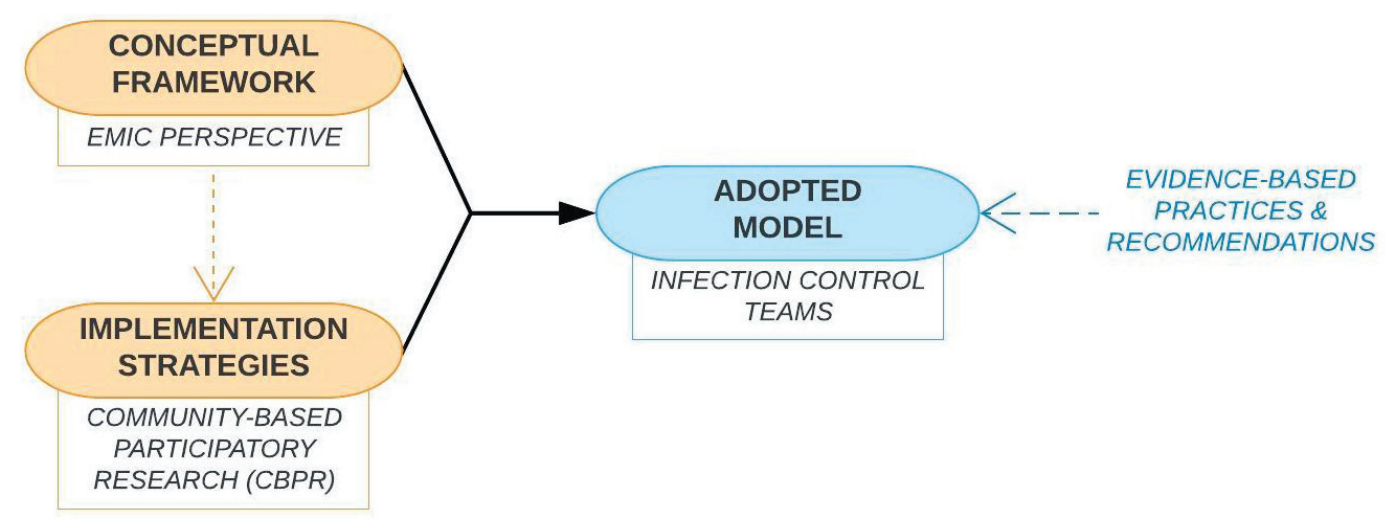

Figure 1. Reframing infection control approaches. 


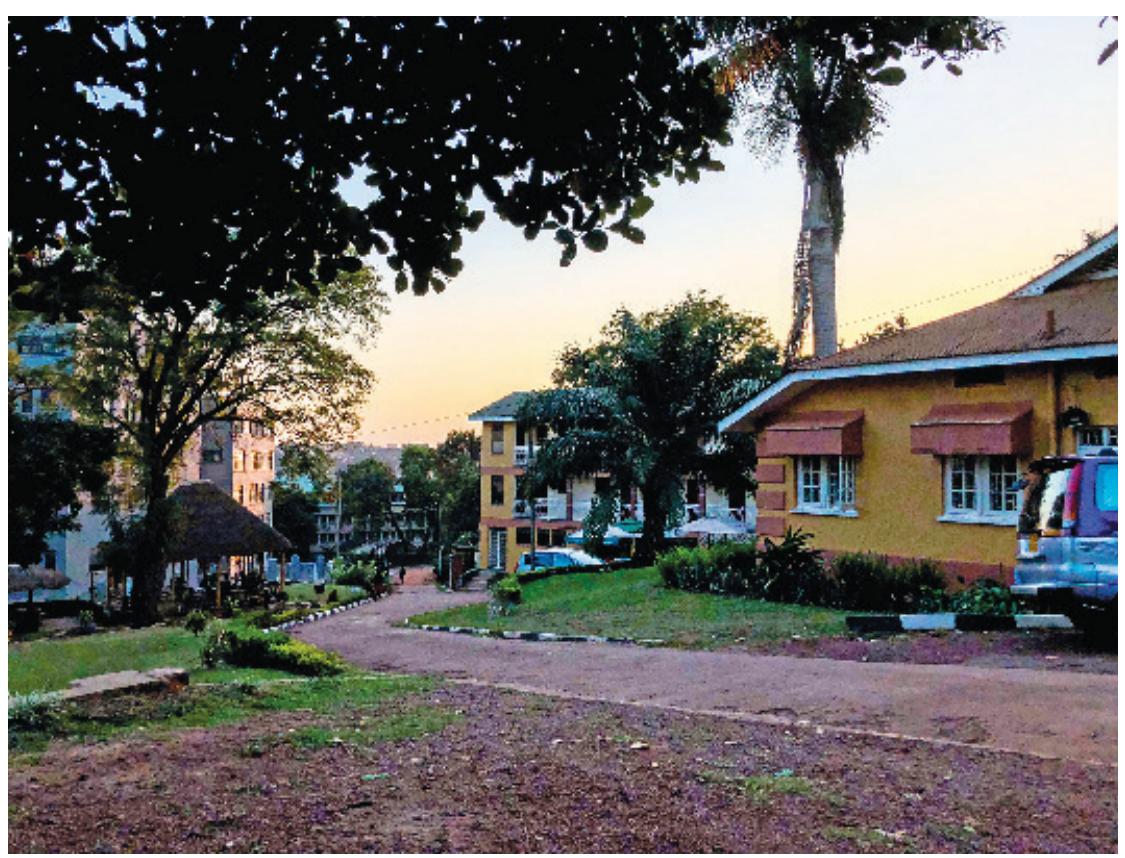

Photo: Guest house at Mulago National Referral Hospital in Kampala, Uganda (from Samantha Sadler's collection, used with permission). searchers and community partners, wherein both maintain the right to provide input on the project with the promise of thoughtful consideration by the other [10]. In this way, CBPR exemplifies the potential efficacy of a local team where the responsibility and authority ultimately rests with the local stakeholders, particularly in terms of project development and implementation [11]. Therefore, researchers should draw lessons from CBPR studies and team models to systematically develop and implement locally driven infection control efforts.

\section{Adapted Model: the infection control team}

Local infection control and prevention programs run by a dedicated team have been identified as important to infection control in low-resource settings [4]. However, the utility of these local teams may be limited by the fixed, "one-size-fits-all" infection control recommendations for LMICs themselves. For example, Storr et al. (2017) noted that a general weakness to the WHO's infection control guidelines (2016) was that the guidelines could not be considered comprehensive due to the infinite number of possible situations diverse health care settings may face [12]. By adhering to models proven effective in other contexts and thereby imposing these external, etic regulations diminishes the uniquely emic potential of a local infection control team. Moreover, this highlights a trend in infection control research of broadly grouping low-resource settings in a way that eclipses inter-setting diversity to the detriment of local communities.

If properly empowered, the local infection control team (one consisting of and directed by local stakeholders) could address these contextual gaps in infection control intervention design and implementation; through constructing their own adapted infection control initiatives, outcomes might improve by nature of greater local relevance. Limited studies have explored systematically increasing local input in infection control efforts. For example, a study conducted in a Brazilian hospital found that by implementing a twice-monthly meeting for local health care workers to discuss their perceptions of and suggestions to improve hand hygiene, compliance to proper hand hygiene increased, and associated infection rates decreased [13] An example of intentionally leveraging the emic perspective in infection control efforts lies in a program implemented by Duke Global Neurosurgery \& Neurology (DGNN) in Kampala, Uganda; the Neurosurgery Infection Control Team (NICT) was developed among Mulago National Referral Hospital (MNRH) neurosurgery ward staff as a case study examining the feasibility and acceptability of a local infection control team in terms of performance effectiveness and attitudinal and behavioral outcomes. Preliminary results suggest a high degree of self-efficacy and empowerment among NICT members, as well as improved infection rates. A more in-depth analysis is forthcoming [14] A study conducted in Columbia used a similar method of guided project development; two separate infection control teams each consisting of local hospital personnel were encouraged to design a post-cesarean infection reduction approach, and each successfully improved clinical outcomes through their different proposed methods [15] Through applying and systematizing such community-based participation, a designated local infection team consisting of local staff familiar with ward norms could systematically facilitate the production and implementation of such locally-informed approaches.

Moreover, the CBPR implementation model explored above may help local infection control teams navigate financial and infrastructural barriers that may hinder their effectiveness. However, such a partnership must be pursued with great caution; the CBPR principle of local stakeholder empowerment must be prioritized to ensure any outside provision of support does not perpetuate historical power differentials and cause undue dependability on external providers. 


\section{CONCLUSION}

An intervention is only as effective as its implementation. As the latter remains a challenge in low-resource settings due to financial, infrastructural and other contextual challenges, explicit consideration must be given to adapting SARS-CoV-2 infection control practices to local contexts. Through implementation strategies prioritizing local stakeholder empowerment (eg, CBPR-derived), the local infection control team model could be feasibly adapted to facilitate locally informed (and thereby emic) infection control approaches.

Few studies have assessed ways in which such local infection control teams might provide the flexibility needed to ensure infection control practices are adopted while ensuring efficacy of the infection control practices in place. Future studies should further investigate the trade-offs between prioritizing local specificity over existing evidence-based practices and outcomes in terms of local stakeholder perspective, infection control rates, and relative sustainability of implementation and change.

SARS-CoV-2 threatens to exacerbate the already disparate burden of HCAIs in low-resource settings, highlighting the urgent need to improve infection control efforts in these facilities. As researchers consider ways to best combat this spread, such approaches must prioritize the emic perspective to maximize local empowerment and representation during this time of global need.

Acknowledgements: We would like to thank the Mulago National Referral Hospital (MNRH) neurosurgery staff for their continued commitment to their communities and collaboration with Duke Global Neurosurgery and Neurology (DGNN) to progress equitable care in global neurosurgery.

Funding: No external funding was obtained for this piece.

Authorship contributions: SS developed the described framework and wrote the manuscript. AF provided mentorship and critical feedback to shape the manuscript.

Competing interests: The authors completed the ICMJE Unified Competing Interest form (available upon request from the corresponding author), and declare no conflicts of interest.

1 Allegranzi B, Nejad SB, Combescure C, Graafmans W, Attar H, Donaldson L, et al. Burden of endemic health-care-associated infection in developing countries: systematic review and meta-analysis. Lancet. 2011;377:228-41. Medline:21146207 doi:10.1016/S0140-6736(10)61458-4

2 Bong C-L, Brasher C, Chikumba E, McDougall R, Mellin-Olsen J, Enright A. The COVID-19 Pandemic: Effects on Low and Middle-Income Countries. Anesth Analg. 2020;131:86-92. Medline:32243287 doi:10.1213/ANE.0000000000004846

3 Nussbaumer-Streit B, Mayr V, Dobrescu AI, Chapman A, Persad E, Klerings I, et al. Quarantine alone or in combination with other public health measures to control COVID-19: a rapid review. Cochrane Database Syst Rev. 2020;4:CD013574. Medline:32267544

4 Vilar-Compte D, Camacho-Ortiz A, Ponce-de-León S. Infection control in limited resources countries: Challenges and priorities. Curr Infect Dis Rep. 2017;19:20. Medline:28401447 doi:10.1007/s11908-017-0572-y

5 Chakkalakal RJ, Cherlin E, Thompson J, Lindfield T, Lawson R, Bradley EH. Implementing clinical guidelines in lowincome settings: A review of literature. Glob Public Health. 2013;8:784-95. Medline:23914758 doi:10.1080/17441692 .2013 .815794

6 Gardam M, Reason P, Gitterman L. Healthcare-associated infections: new initiatives and continuing challenges. Healthc Q. 2012;15:36-41. Medline:22874445 doi:10.12927/hcq.2012.22843

7 Berry JW. Imposed Etics_Emics—Derived Etics: The Operationalization of a Compelling Idea. Int J Psychol. 1989;24:72135. doi:10.1080/00207598908246808

8 Anderson D. Multicultural Group Work: A Force for Developing and Healing. J Spec Group Work. 2007;32:224-44. doi:10.1080/01933920701431537

9 Dramowski A, Cotton MF, Whitelaw A. Surveillance of healthcare-associated infection in hospitalised South African children: Which method performs best? S Afr Med J. 2016;107:56-63. Medline:28112093 doi:10.7196/SAMJ.2017. v107i1.11431

10 Ross LF, Loup A, Nelson RM, Botkin JR, Kost R, Smith GR, et al. The challenges of collaboration for academic and community partners in a research partnership: Points to consider. J Empir Res Hum Res Ethics. 2010;5:19-31. Medline:20235861 doi:10.1525/jer.2010.5.1.19

11 Lazarus S, Taliep N, Bulbulia A, Phillips S, Seedat M. Community-based participatory research a low-income setting: An illustrative case of study. J Psychol Afr. 2012;22:509-16. doi:10.1080/14330237.2012.10820562 
12 Storr J, Twyman A, Zingg W, Damani N, Kilpatrick C, Reilly J, et al. Core components for effective infection prevention and control programmes: new WHO evidence-based recommendations. Antimicrob Resist Infect Control. 2017;6:6. Medline:28078082 doi:10.1186/s13756-016-0149-9

13 Marra AR, Guastelli LR, de Araújo CMP, dos Santos JLS, Lamblet LCR, Silva M, et al. Positive deviance a new strategy for improving hand hygiene compliance. Infect Control Hosp Epidemiol. 2010;31:12-20. Medline:19925270 doi:10.1086/649224

14 Sadler S, Fuller A. Infection Control from an Emic Perspective: Forming and Evaluating a Local Infection Control Team in a Low-Resource Neurosurgery Ward. Unpublished Manuscript, Duke University, Durham, NC.

15 Weinberg M, Fuentes JM, Ruiz AI, Lozano FW, Angel E, Gaitan H, et al. Reducing Infections Among Women Undergoing Cesarean Section in Colombia by Means of Continuous Quality Improvement Methods. Arch Intern Med. 2001;161:2357-65. Medline:11606152 doi:10.1001/archinte.161.19.2357

\section{Correspondence to:}

Samantha J Sadler

Duke University, Bachelor's of Science, Neuroscience \&

Global Health (2019). Harvard Medical School

(Expected 2023)

30 Garrison Street, Unit 30-105

Boston, Massachusetts, 02116

USA

Samantha_sadler@hms.harvard.edu 